\title{
A method for photo-identification of turtles of the Phrynops williamsi species
}

\author{
Guilherme Oecksler Bertoldi $^{1}$, Aurélio Faustino Hoppe ${ }^{1}$ \\ ${ }^{1}$ Department of Computer Science \\ Regional University of Blumenau (FURB) - Blumenau, SC - Brazil
}

\begin{abstract}
This paper presents a method for photo-identification of Phrynops williamsi turtles. This identification is performed using shape descriptors which computationally represent the horseshoe-shaped and circular-shaped bands that can be found on the ventral surface of the turtle's head. The input image is converted to grayscale, binarized, filtered with morphologic operations, segmented based on the contours of the object and the components are selected based on their geometric characteristics. With the extraction of these characteristics, the method calculates de Fourier Descriptors and create a unique identifier used to identify the turtle from the input image. Results show that the presented method has reached a success rate of $85.71 \%$ in intra-class comparison and $85.17 \%$ in inter-class comparison.
\end{abstract}

\section{Introduction}

The aim for monitoring animal specimens is to understand their age group, density, sex ratio and the survival rates of a certain animal specimen [Spier et al. 2014]. By monitoring it is also possible to determinate the variation in the number of individuals of the species during a period of time. When well planned, it even allows the biologists to assess the impact of a particular management practice and conservation, diseases, hunt and habitat conservation of a species [Balestra et al. 2016].

In the chelonians, the monitoring is realized by marking the captured specimens [Balestra et al. 2016]. The most utilized chelonians' marking technique is conducting incisive markings made in their shell, featuring an invasive method. This type of marking causes stress to the animals and, in eventual circumstances, it results in their death due to the injuries generated [McDiarmid et al. 2012]. It is a low cost method however the cuts may disappear due to natural regeneration. The incisions are performed using hacksaws (Figure 1a) and it is recommended that they have a square or rectangular shape (Figure $1 b)$, preventing them from being confused with possible bites from another animal. The captured specimens are individualized using a numerical codification generated by the disposition of the incisions in the shells (Figure 1c) [Balestra et al. 2016].
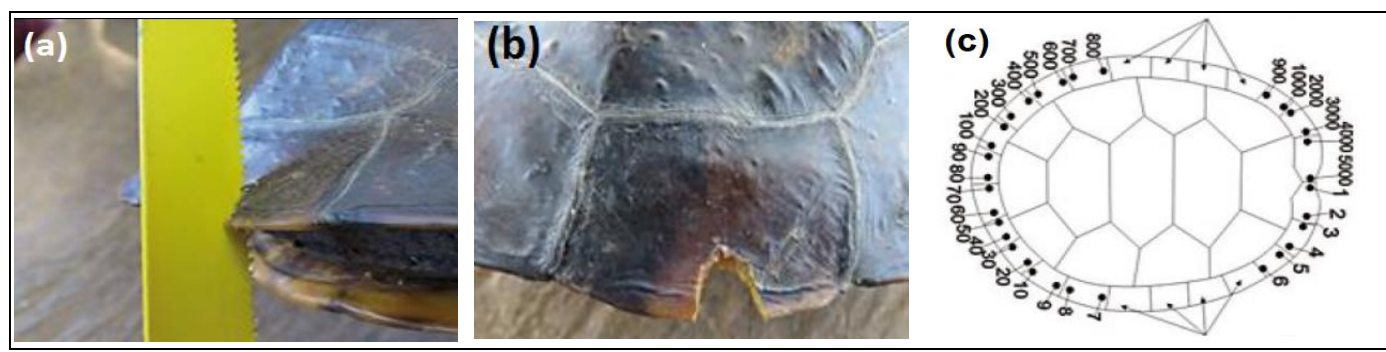

Figure 1. Chelonians' marking method: (a) utilized hacksaws, (b) cut shape, (c) numerical scheme marking of shells. 
In this scenario, the objective of this study is the development of a new computational method based on the characteristics of the shape of stripes for the identification of turtles of the Phrynops williamsi species, through a non-invasive method. The turtles of the Phrynops williamsi species, commonly known as William's South American side-necked turtle, are diurnal animals that live in large rivers with muddy or rocky shores [Vogt et al. 2015]. They inhabit areas with altitude less than 500 meters from the east coast of Santa Catarina and Rio Grande do Sul, as well as half of Uruguay [Rhodin and Russel 1983].

Studies directly related to the object of this study were not found however, we list some studies that bore more resemblance to, or that utilize techniques which can possibly be used to try to create a unique identifier for turtles of the Phrynops williamsi species. [Baboo and Vigneswari 2014] presented in their studies, a tool for the identification of particular species of the Olive ridley sea turtle in the MATLAB environment. The objective of the proposed tool is to make the identification of the turtles through the extraction of their features by an input image. [Beugeling and Branzan-albu 2014] proposed an algorithm for automatic and individual identification of the turtles based on the images of their plastrons. The proposed algorithm aims to decrease the time used by scientists in a non-invasive identification of turtles during the monitoring of a particular population. [Pic4Turtle 2016] is an application for identification of species of a sea turtle through a mobile device. The user must send an image of the turtle obtained through the device camera or the gallery of the device itself for identification. If the species is identified, some information such as if it is in extinction, its food, their habitat, size, weight and some curiosities, is returned.

\section{Photo-identification method}

In this section, we present a method for photo-identification of turtles of the Phrynops williamsi species, with the objective of assisting biologists and researchers in the identification of this species using a non-invasive method. The proposed method is divided into three main steps: Image Segmentation, Stripes Selection and Turtle Rating. The next section describes the Image Segmentation step.

\subsection{Image segmentation}

The segmentation process should segment the objects of interest from the image backgroun. This process defines the eventual success or failure of image applications, being one of the most costly process of this type. Figure 2 illustrates a diagram of this step on the proposed method. Initially, the original colorful image (Figure 3a) is converted to grayscale (Figure $3 \mathrm{~b}$ ), once the classification of the turtles uses only the format of the stripes, the color being irrelevant in the process. In this step the objective is to isolate the objects of interest from the image background. Figure 2 shows the steps that make up the image segmentation process.

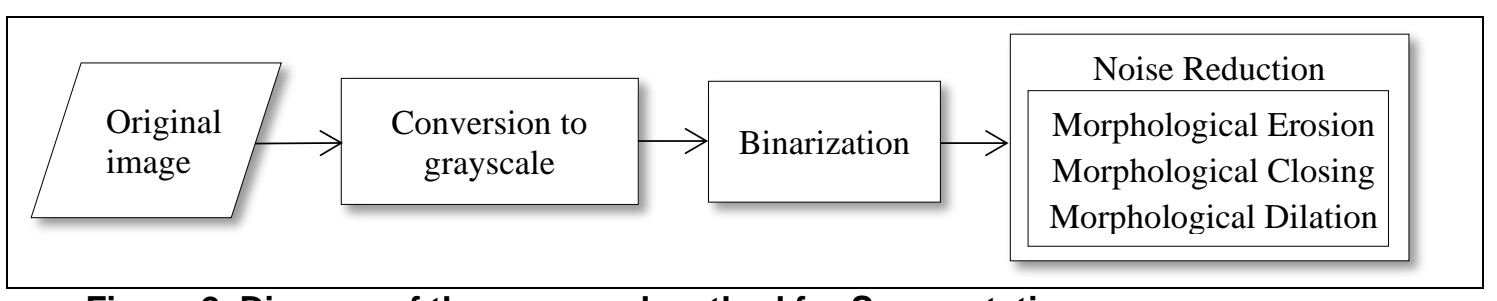

Figure 2. Diagram of the proposed method for Segmentation. 
Here, Threshold technique is utilized to segment the image. This is a binarization technique that transforms the pixels image, from gray scale to black and white pixels. On this method all pixels images are analyzed and compared with a threshold value. In the proposed method a Threshold value of 72 is utilized, a value that has shown better results in the performed tests. As previously discussed, the result of this process is the conversion of the grayscale image (Figure $3 b$ ) to a black and white image (Figure 3c).
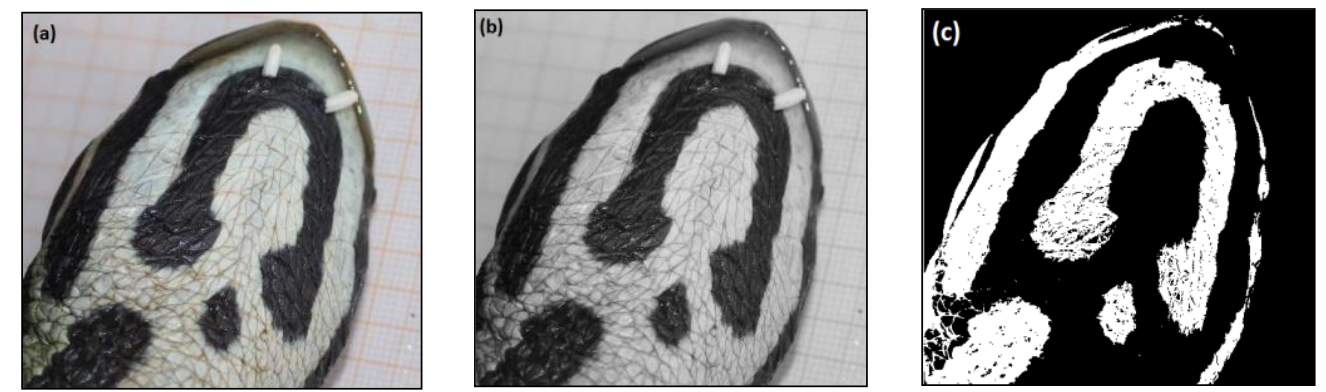

Figure 3. Result of the image segmentation process: (a) original image, (b) grayscale image, (c) binarized image.

Figure 3 shows the binarization process resulting in an image full of imperfections and noises, making it difficult to correctly identify the elements which represent the image. In order to correct them, three types of morphological operations are applied to make these more homogeneous elements. First, the morphological erosion filter is applied with a $3 \times 3$ size Structural Element (SE), as shown in Equation. (1).

$$
\mathrm{A} \ominus \mathrm{B}=\left\{\mathrm{z} \mid(\mathrm{B})_{\mathrm{z}} \subseteq \mathrm{A}\right\}
$$

Then the closing filter is applied with a $12 \times 12$ size SE, as shown in Equation. (2).

$$
\mathrm{A} ¥ \mathrm{~B}=(\mathrm{A} \oplus \mathrm{B}) \ominus \mathrm{B}
$$

Finally, the morphological dilation operation is applied with a $3 \times 3$ size SE, as shown in Equation. (3).

$$
\mathrm{A} \oplus \mathrm{B}=\left\{\mathrm{z} \mid(\widehat{\mathrm{B}})_{\mathrm{z}} \cap \mathrm{A} \neq \varnothing\right\}
$$

Where A represents the segmented input image and B is the SE used in the operation. Figure 4 shows the results achieved in each of the morphological operations.

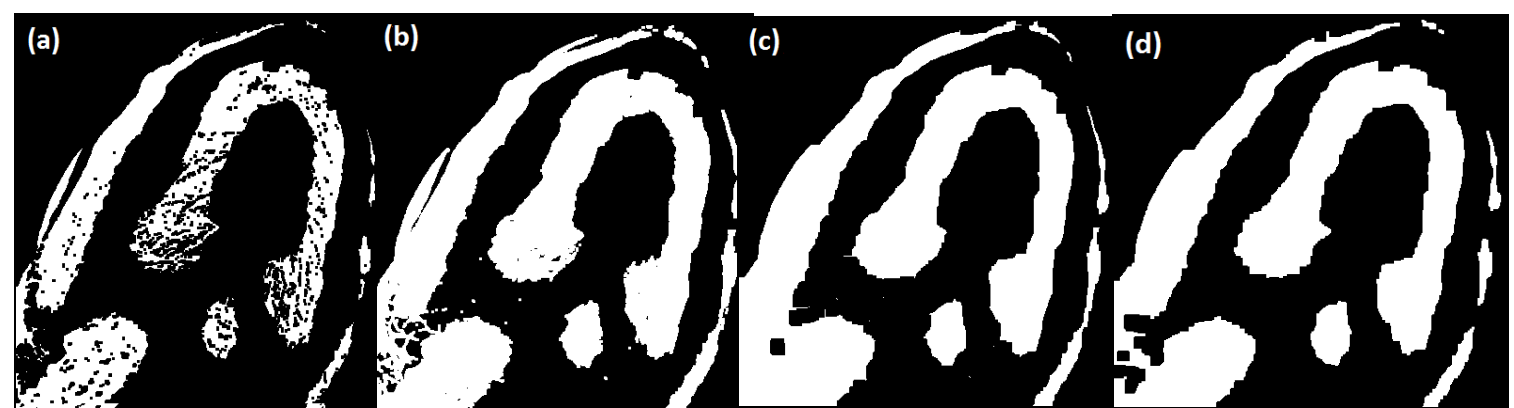

Figure 4. Result of the image noise removal process: (a) erosion operator, (b) dilation operator, (c) closing operator, (d) filtered image.

The erosion operator performs the thinning of image objects, causing the elimination of stray pixels (Figure 4a). Similarly to the erosion filter, the dilatation filter performs thickening of the objects resulting in filling in the gaps that may exist in them (Figure 4b). The closing filter (Figure 4c) in turn, is the combined application of the dilatation filter followed by the application of the erosion filter. After these operations, 
the elements become more homogeneous and integral (Figure 4d). This noise removal facilitates the extraction and selection of the components that relate to the stripe in the horseshoe format and in the circular-shaped stripe.

\subsection{Stripes extraction and selection}

In the stripes selection process, the related components of the image are extracted, then the horseshoe-shaped stripe and the circular-shaped stripe are identified. Figure 5 shows the extraction and selection of stripes diagram.

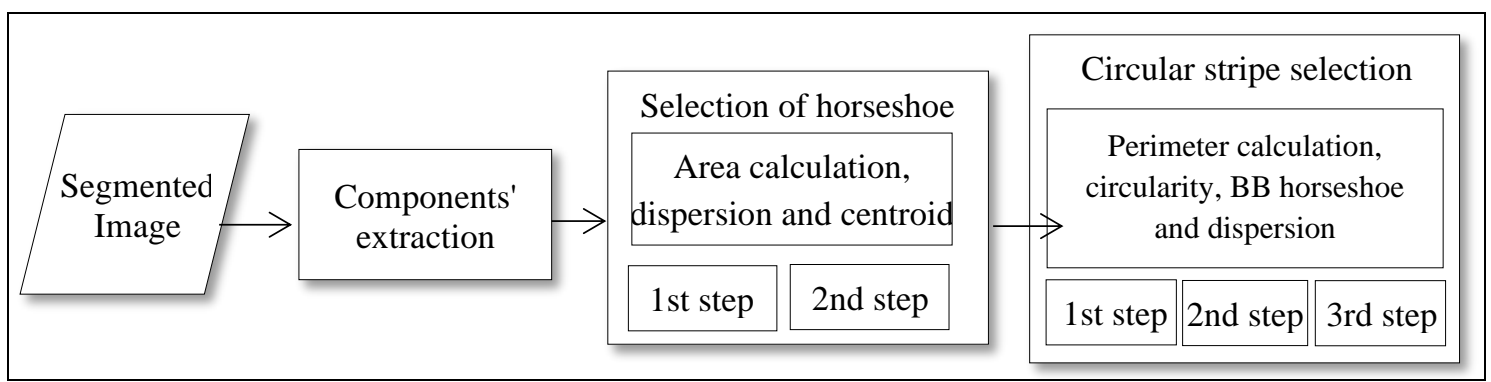

Figure 5. Diagram of the selection step of the stripes for the proposed method

The extraction of the components is performed by the analysis of the contour characteristics of the elements that compose the image. For this, the algorithm proposed by [Suzuki 1985] was used. The application of this technique in a segmented image, results in an array containing the contour pixels of each of the objects in image. Figure 6 graphically illustrates the result of this extraction where the extracted components were colored with distinct colors for illustration purposes.

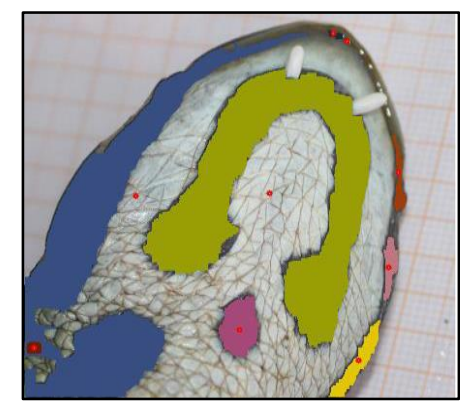

Figure 6. Result of extraction of components through contour identification

With the components extracted and stored in an array list, the next step is to identify the component in respect to the stripe-shaped horseshoe. The horseshoe selection step is responsible for identifying which of the components extracted in the previous step concerns the horseshoe-shaped stripe. In order to carry out this selection, the following characteristics of the components were used: area, centroid and dispersion. Centroid is the center of mass of the object, obtained by averaging the sum of the object contour coordinates, as shown in Equation (4).

$$
M=\frac{\sum_{n=0}^{N-1} u(n)}{N}
$$

Where, $u(n)$ represents the $n$th pixel coordinate function of the contour and $N$ the number of pixels of the object. Dispersion is the measure indicating how irregular the form of a 2D object is shown in Equation (5). Proposed by [Nixon and Aguado 2002], the dispersion is measured by the ratio between the largest and smallest distance between the centroid and the contour of the object.

$$
\text { dispersion }=\frac{\max \left(\sqrt{\left(x_{i}-\bar{x}\right)^{2}+\left(y_{i}-\bar{y}\right)^{2}}\right)}{\min \left(\sqrt{\left(x_{i}-\bar{x}\right)^{2}+\left(y_{i}-\bar{y}\right)^{2}}\right)}
$$


Where, $(\bar{x}, \bar{y})$ represent the coordinate of the centroid of the object. To obtain this rate, all pixels of the contour are analyzed in order to find the largest (max) and smallest (min) between the centroid and the contour of the object. The horseshoe selection was divided into two steps, the second step is only realized when the stripe is not identified in the first step. In the first step, in order to select the horseshoe, the following premises are observed:

- The horseshoe-shaped component tends to be the second largest component in relation to the area;

- The distance between the centroid of the horseshoe and the farthest point of the contour, tends to be smaller than the distance of the largest component.

These two premises should be checked for all image components. For this purpose, in the proposed method the following steps are performed:

- Create a list with the area of all components;

- Sort it in descending order and keep the first 3 components (Figure 7a);

- Create a new list with the quotient of the area from the distance of the centroid to the farthest point of the contour (Figure 7a, blue lines).

After performing these three steps, the first component of the list will be considered the horseshoe that will be used on the identification of the tortoise, if its area is $50 \%$ larger than the area of the second component. Otherwise, the area and dispersion of the remaining components in the list are checked in the second step.
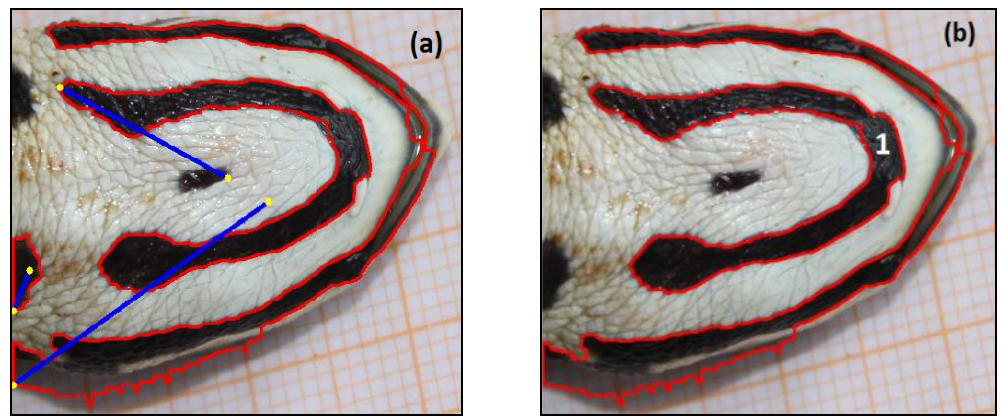

Figure 7. Result of the stripe selection in horseshoe format: (a)step one, (b)step two.

In area verification and dispersion, the two largest components must have a very similar area, and the dispersion of the horseshoe-shaped component tends to be smaller than the dispersion of the other component in the list. The following steps are performed for the three remaining components of the first step:

- Remove the third component of the list;

- Create a new list containing the sum of the component dispersion with its area and sort it in ascending order.

After performing these two steps, the first element of the list is considered to be the horseshoe-shaped stripe, because it has smaller area and less dispersion. Figure $7 \mathrm{~b}$ shows the result of the second step, the horseshoe-shaped component being identified by the digit " $1 "$.

With the selected horseshoe-shaped stripe, the next step is to identify the component that represents the stripe in a circular format. This stripe, when present in the tortoises, is usually located near the horseshoe. For this step, the perimeter, the area and the circularity of the components are used. Circularity is the measure that indicates how circular the shape of an object is. This measure ranges from 0 to 1 , and the closer to 1 , the more the object resembles a circle. The circularity is measured according to Equation (6). 


$$
\text { roundness }=\frac{4 \pi A}{p^{2}}
$$

Where, A represents the area of the object and $p$ the perimeter of the object. The selection of the circular-shaped stripe is divided into three steps, where the components that do not meet the condition of each one, are eliminated. The first step works with the following premises on the circular-shaped stripe component:

- The circular-shaped stripe tends to be of circular format with a degree of circularity greater than 0.45 ;

- The circular-shaped stripe must have half of the horseshoe-shaped stripe perimeter;

- At least one of the points of the circular-shaped stripe tends to be inside the Bounding Box (BB) of the horseshoe-shaped stripe.

For validation of these three assumptions, the checking below must be performed for all components of the image, except the horseshoe-shaped component:

- Check whether the perimeter of the component is less than $50 \%$ of the perimeter of the horseshoe;

- Check whether the area of the component is greater than $2 \%$ of the area of the horseshoe;

- Check whether the degree of circularity of the component is greater than 0.45 .

Figure 8a illustrates the execution of this step. Next to the components that meet the three imposed conditions is the information of perimeter (P), area (A) and circularity $(\mathrm{R})$ of each of one. The components that meet the three validations performed in the first step should move to the second step of the selection, where the following steps are performed:

- The centroid of the components is calculated (Figure 8b, yellow dots);

- Check for at least one point inside the BB of the horseshoe (Figure 8b, blue box);

- The distances between the centroids are calculated (Figure 8b, blue lines);

- If the verification of the second step is accurate, the distance calculated in this step is reduced by $30 \%$. Finally, the list is sorted in an ascending order.
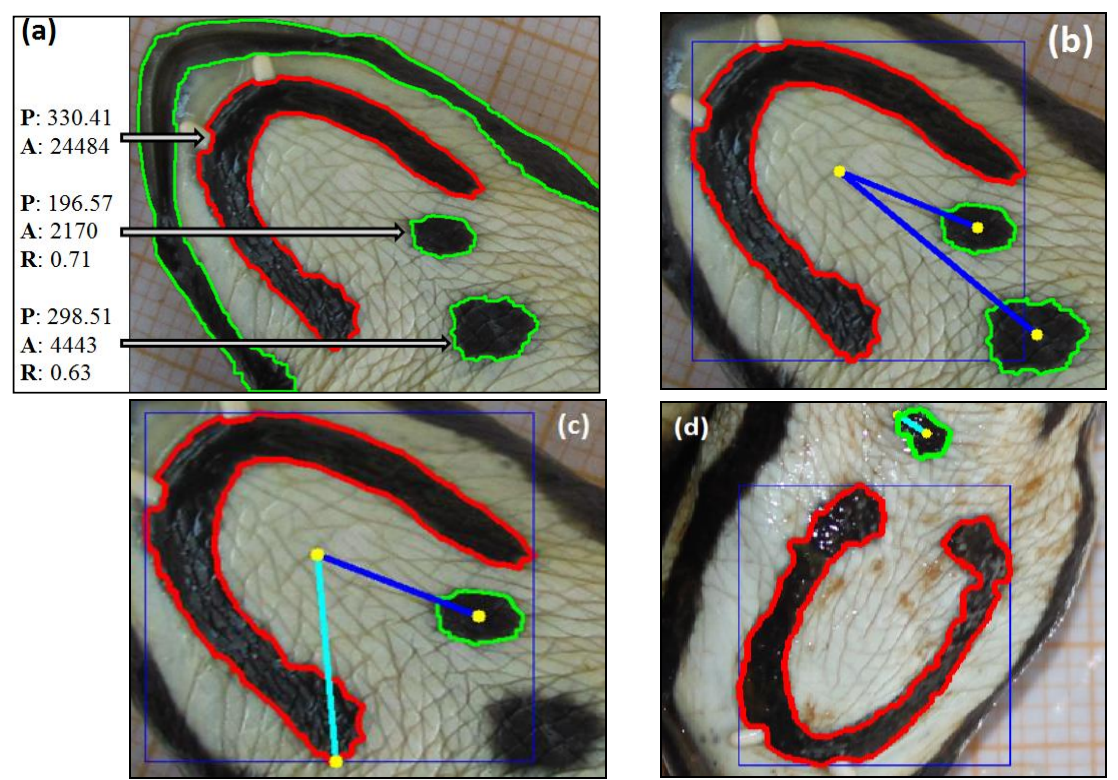

Figure 8. Result of stripe selection in circular format: (a) first step, (b) second step 
At the end of the selection of the second step, it is assumed that the first item in the list concerns the component of the circular stripe. As stated before, some tortoises do not have this stripe, so in the third step the first item must meet the following conditions to be considered the circular stripe:

- Have dispersion less than eight;

- The distance from the centroid to the centroid of the horseshoe (Figure 8c, dark blue line) should be less than $1.5 \mathrm{x}$ the distance between the centroid of the horseshoe to the point of the distal contour (Figure 8c, light blue line);

- The distance between the centroid and the nearest contour point must be greater than five or at least one of the points must be within the BB of the horseshoe (Figure 8d, light blue line).

If the first item of the list satisfies the three conditions above, it is considered the circular stripe otherwise it is assumed that the tortoise does not have this stripe. The following section describes the classification step, where the classification and identification of the tortoise will be done by means of a unique identifier also created in this step.

\subsection{Tortoise classification}

In the classification stage the identification of the tortoises is done. Here the shape descriptors of the horseshoe-shaped and the circular-shaped stripe are calculated by the Farthest Point Distance form signature. From these descriptors the Fourier series is obtained, used here as a unique identifier for the identification of tortoises. Figure 9 show the diagram of the method proposed in this step.

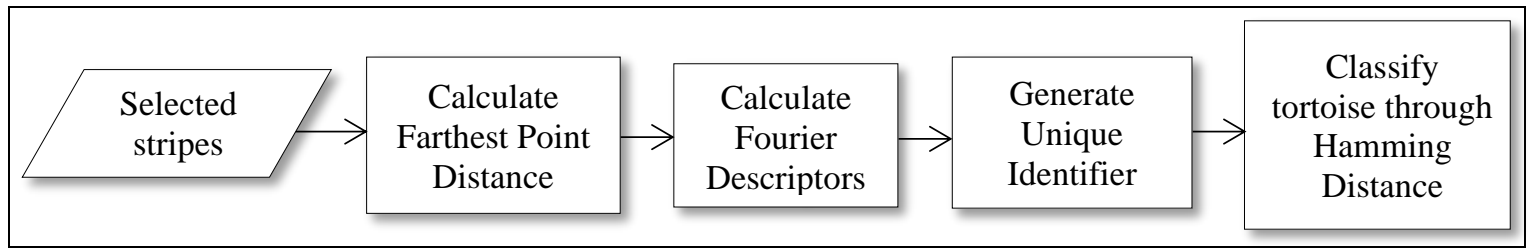

Figure 9. Diagram of the Classification step of the proposed method

In the proposed method, the use of the shape signature, Farthest Point Distance (FPD) was chosen instead of classical radial distance because it showed better results. According to the authors, the approach adopted by the FPD signature considers the corners distances of the object, a fundamental feature in the human view, disregarded in most existing techniques [El-ghazal, Basir and Belkasim 2009].

In the shape signature presented by [El-ghazal, Basir and Belkasim 2009], called Farthest Point Distance, each of the contour points of the analyzed object is represented by two distances. The first is the distance from the analyzed point to the object centroid and the second is the distance from this centroid to the point of the most distant contour of the analyzed point. FPD is defined as:

$$
F P D(i)=\sqrt{\left(\left[x_{i}-\bar{x}\right]^{2}+\left[y_{i}-\bar{y}\right]^{2}\right)}+\sqrt{\left[x_{i f p}-\bar{y}\right]^{2}+\left[y_{i f p}-\bar{y}\right]^{2}}
$$

Where, $\left(x_{i}, y_{i}\right)$ represents the coordinates of the contour points of the analyzed object, $(\bar{x}, \bar{y})$ represents the coordinates of the centroid of the object e $\left(x_{\text {ifp }}, y_{\text {ifp }}\right)$ the point coordinates of the contour farthest from the $\mathrm{i}$-th analyzed point. The FPD shape signature is used in the calculation of the Fourier Descriptors.

Flat-shape discrimination is one of the most familiar and fundamental problems in pattern recognition. Fourier descriptors are widely used in the description of flat-shape 
objects. These descriptors are obtained by means of the Discrete Fourier Transform, which is calculated through the sum of sine and cosines, and each of the values is multiplied by a different coefficient. DFT used in this work is defined as:

$$
F(u)=\sum_{x=0}^{M-1} f(x) e^{\frac{-j 2 \pi u x}{M}} \quad u=0,1,2, \ldots, M-1
$$

Where, $M$ represents the quantity of values of the transform, $\mathrm{x}$ the $\mathrm{x}$-th value of the transform and $\mathrm{u}$ the $\mathrm{u}$-th descriptor. The DFT is computed as follows. For each of the series values, all the contour pixels are traversed and their FPDs are obtained. These distances are multiplied by a coefficient other than theta defined by $-j 2 \pi u x / M$. Finally, each value of the series is represented by a complex discrete function

$$
s(k)=x(k)+j y(k)
$$

Where, $\mathrm{x}$ represent the real part and $\mathrm{y}$ the imaginary part of the descriptor. The invariance to translation of the descriptors is obtained by zeroing the first descriptor of the series: $\mathrm{F}(\mathrm{u})=0$. The invariance to rotation is obtained by keeping only the magnitudes of the values of the series: $F(u)=|F(u)|$. The division of the descriptors by the second descriptor of the list makes the series invariant to scale: $F(u)=$ $|\mathrm{F}(\mathrm{u})| /|\mathrm{F}(2)|$.

The unique identifier used in the classification of the proposed method is given through the Fourier series, which is calculated on the horseshoe-shaped and circularshaped stripes. Unique identifiers generated from distinct images of the same tortoise have similarity in all their values. Whereas an identifier generated from the image of a distinct tortoise presents a difference in most values. The low frequency descriptors accumulate more energy possessing greater variation between the values, enough to represent the general form of the objects.

The classification of the identifiers is performed through the Hamming distance, where all the values of the arrays are compared and their differences are accumulated. This distance of the identifier of the informed tortoise is calculated based on all the identifiers stored in the database. In the sum of distances, only differences greater than $10 \%$ of the highest compared value are considered. In the classification, a threshold of 12 is used, that is, the compared tortoises are considered different if the result of this distance is greater than 12 otherwise they are considered the same tortoises.

\section{Experimental results and discussions}

To validate the proposed method, tests were performed on a base composed by 53 images of 26 different tortoises. For the images, digital cameras and typical field procedures were used to obtain ecological data. The images were obtained from the lower part of the animals and the region of interest (lower part of the head) was selected manually.

In the performed test, 23 descriptors were used in the horseshoe-shaped stripe and 6 in the circular-shaped stripe, where intra-class and inter-class comparisons were made. In intra-class comparisons the image of a tortoise is compared with the other images of the same tortoise. Table 1 shows the results of the performed tests. In this table the sample quantities are displayed, that is, the number of images for each tortoise of the dataset. It also shows the number of performed comparisons, the number of correct comparisons and the percentage of correctness for each type of comparison, intra-class and inter-class. 


\begin{tabular}{|c|c|c|c|c|c|c|c|}
\hline Individuals & $\begin{array}{c}\text { Samples } \\
\text { No. }\end{array}$ & $\begin{array}{c}\text { Intra-class } \\
\text { Ratings No. }\end{array}$ & $\begin{array}{c}\text { Correct } \\
\text { intra-class } \\
\text { ratings No. }\end{array}$ & $\begin{array}{l}\text { Intra-class } \\
\text { correctness } \\
\text { percentage }\end{array}$ & $\begin{array}{c}\text { Inter-class } \\
\text { Ratings No. }\end{array}$ & $\begin{array}{c}\text { Correct } \\
\text { inter-class } \\
\text { ratings No. }\end{array}$ & $\begin{array}{l}\text { Inter-class } \\
\text { correctness } \\
\text { percentage } \\
\end{array}$ \\
\hline PW01 & 1 & - & - & - & 52 & 50 & $96,15 \%$ \\
\hline PW02 & 3 & 6 & 6 & $100,00 \%$ & 147 & 147 & $100,00 \%$ \\
\hline PW03 & 1 & - & - & - & 48 & 48 & $100,00 \%$ \\
\hline PW04 & 1 & - & - & - & 47 & 31 & $65,96 \%$ \\
\hline PW05 & 1 & - & - & - & 46 & 27 & $58,70 \%$ \\
\hline PW06 & 1 & - & - & - & 45 & 45 & $100,00 \%$ \\
\hline PW07 & 1 & - & - & - & 44 & 28 & $63,64 \%$ \\
\hline PW08 & 1 & - & - & - & 43 & 43 & $100,00 \%$ \\
\hline PW09 & 1 & - & - & - & 42 & 30 & $71,43 \%$ \\
\hline PW10 & 4 & 12 & 10 & $83,33 \%$ & 152 & 146 & $96,05 \%$ \\
\hline PW11 & 3 & 6 & 6 & $100,00 \%$ & 105 & 104 & $99,05 \%$ \\
\hline PW12 & 6 & 30 & 28 & $93,33 \%$ & 174 & 109 & $62,64 \%$ \\
\hline PW13 & 1 & - & - & - & 28 & 25 & $89,29 \%$ \\
\hline PW14 & 2 & 2 & 2 & $100,00 \%$ & 52 & 31 & $59,62 \%$ \\
\hline PW15 & 1 & - & - & - & 25 & 16 & $64,00 \%$ \\
\hline PW17 & 3 & 6 & 6 & $100,00 \%$ & 66 & 65 & $98,48 \%$ \\
\hline PW18 & 2 & 2 & 2 & $100,00 \%$ & 40 & 32 & $80,00 \%$ \\
\hline PW19 & 4 & 12 & 10 & $83,33 \%$ & 64 & 55 & $85,94 \%$ \\
\hline PW20 & 1 & - & - & - & 15 & 11 & $73,33 \%$ \\
\hline PW21 & 3 & 6 & 6 & $100,00 \%$ & 36 & 35 & $97,22 \%$ \\
\hline PW22 & 2 & 2 & 2 & $100,00 \%$ & 20 & 20 & $100,00 \%$ \\
\hline PW23 & 2 & 2 & 0 & $0,00 \%$ & 16 & 16 & $100,00 \%$ \\
\hline PW24 & 1 & - & - & - & 7 & 7 & $100,00 \%$ \\
\hline PW25 & 3 & 6 & 0 & $0,00 \%$ & 12 & 8 & $66,67 \%$ \\
\hline PW26 & 1 & - & - & - & 3 & 3 & $100,00 \%$ \\
\hline PW28 & 3 & 6 & 6 & $100,00 \%$ & 0 & 0 & - \\
\hline Total & 53 & 98 & 84 & $85,71 \%$ & 1329 & 1132 & $85,17 \%$ \\
\hline
\end{tabular}

Table 1. Intra-class and inter-class comparisons results

From Table 1, it can be noticed that 98 intra-class comparisons were made, of which 84 were correctly classified by the proposed method, i.e., it indicated that the tortoises of the two images were the same. From these results, we conclude that the proposed method obtained an intra-class accuracy rate of $85.21 \%$. Finally, it can be observed that 1329 inter-class comparisons were made, of which 1132 were correctly identified, that is, the proposed method showed that the tortoises of the two images compared were not the same. With these values, it is possible to affirm that the inter-class accuracy rate of the method is $85.17 \%$.

\section{Conclusion and Future Works}

We have proposed the development of a new method for recognition of tortoises of the Phrynops williamsi species via digital images. From the obtained results it can be determined that the proposed method is a viable alternative to accomplish the recognition of tortoises. Where, in the intra-class comparisons a top rate of $85.71 \%$ and in the interclass comparisons a rate of $85.17 \%$, were obtained.

Finally, it is concluded that the proposed method can be used by researchers and field biologists who are studying this kind of tortoise, assisting them in the identification of these tortoises utilizing a non-invasive method. In addition, it can still be used as a basis for other studies focusing the identification of other species, and/or turtles. As future work, we suggested studies to find a more effective identification method. For example: improving the method of segmentation to enable identification of tortoises that have the horseshoe-shaped stripe attached to other stripes of the tortoise head; New methods that 
allow the identification of other species of tortoises. The accuracy rate of the algorithm can be increased by improving the segmentation method to remove external noises such as the presence of reflection on the horseshoe-shaped stripe, in which angle the image was taken and the lack of sharpness of some images.

\section{References}

Baboo, Santhosh; Vigneswari, A. R. J. Identification of Olive Ridley Turtle Using Feature Extraction, in: 2014 Int. Conf. Intell. Comput. Appl., IEEE, 2014: pp. 69-72.

Balestra, Rafael Antônio. Roteiro para Inventários e Monitoramentos de Quelônios Continentais, Biodiversidade Bras. 6 (2016) 114-152.

Beugeling, Trevor; Branzan-Albu, Alexandra Computer Vision-Based Identification of Individual Turtles Using Characteristic Patterns of Their Plastrons, in: 2014 Can. Conf. Comput. Robot Vis., IEEE, 2014: pp. 203-210.

El-ghazal, Akrem; Basir, Otman; Belkasim, S. Farthest point distance: A new shape signature for Fourier descriptors, Signal Process. Image Commun. 24 (2009) 572-586.

McDiarmid, Roy et al. Reptile biodiversity: Standard methods for inventory and monitoring, University of California Press, 2012.

Nixon, Mark; Aguado, Alberto. Elsevier Science Publishers., Feature extraction and image processing, Newnes, 2002.

Pic4Turtle, (2016). www.pic4turtle.com.

Rhodin, Anders G. J; Russel, Anthony. Description of Phrynops williamsi, a new species of chelid turtle of the South American Phrynops geoffroanus complex, in: Adv. Herpetol. Evol. Biol. Essays Honor Ernest E. Williams, Cambridge, Mass. :Museum of Comparative Zoology, 1983: pp. 58-73.

Spier, Edson Fernando et al. Registro de Phrynops williamsi (Rhodin \&amp; Mittermeier, 1983) no rio do Peixe, centro-oeste de Santa Catarina, Brasil, Brazilian J. Biosci. 12 (2014) 56-57.

Suzuki, Satoshi, Topological structural analysis of digitized binary images by border following, Comput. Vision, Graph. Image Process. 30 (1985) 32-46.

Vogt, Richard Carl. Avaliação do Risco de Extinção de Phrynops williamsi Rhodin \& Mittermeier, 1983 no Brasil, (2015). 